\title{
FIELD DEPENDENCE OF CURRENTS*
}

\author{
S. RAI CHOUDHURY, Y. TOMOZAWA and Y. P. YAO \\ Randall Laboratory of Physics, \\ The Universily of Michigan, Ann Arbor, \\ Michigan 48104
}

Received 9 June 1969

\begin{abstract}
Arguments are presented to show that in a theory with spin $\frac{1}{2}$, vector and axial-vector fields, the vector current in general depends upon the axial-vector field also in addition to the dependence on the vector field. The exact dependence is worked out on quite general grounds. We point out, however, that there are inconsistencies in the approach in which use is made of the definition of field dependent current and the usual canonical commutation relations of vector and axial-vector fields.
\end{abstract}

\section{INTRODUCTION}

It has recently been pointed out by Adler [1], that in studying the matrix element of the axial-vector current between vacuum and a $2 \gamma$ state $\left\langle\gamma \gamma\left|j_{\mu} 5(0)\right| 0\right\rangle$, one obtains results in disagreement with the ones obtained through formal manipulations of the field equations. The discrepancy can be traced back to the very singular nature of the product of fermion field operators at the same space-time point [2]. If one defines however all currents as limits of products of fermion fields at slightly separated points, then of course the definition is non-unique in the sense that one can include in the definition dependence of the current $j_{\mu} 5(x)$ on the vector-fields, e.g., through a factor like $\exp \left[i e \int_{x-\frac{1}{2} \epsilon}^{x+\frac{1}{2} \epsilon} A_{\mu}(\xi) \mathrm{d} \xi^{\mu}\right]$, such that the current formally looks like its conventional definition as $\epsilon \rightarrow 0$. The matrix element $\left\langle\gamma \gamma\left|j_{\mu} 5(0)\right| 0\right\rangle$ however is critically dependent on such factors, and in general this matrix element satisfies neither the transversality condition with respect to the external photon momenta, nor the PCAC condition on $j_{\mu} 5(x)$. Definite choices of the dependence of $j_{\mu} 5(x)$ on $A_{\mu}(x)$ exists for which the transversality condition or the PCAC condition can be singly satisfied but they come out to be mutually incompatible. The main part of this note is devoted to a corresponding study of the dependence of the vector-current on fields, when there exists in the theory in addition to the photon field $A_{\mu}(x)$,

* Work supported in part by the US Atomic Energy Commission. 
an axial field $a_{\mu}(x)$ also. The dependence of $j_{\mu}(x)$ on $A_{\mu}(x)$ has been studied by Boulware and Deser [3], but we show here by studying the matrix element $\left\langle\gamma\left|j_{\mu}(0)\right| a\right\rangle$ and also from other general considerations that $j_{\mu}(x)$ has a non-trivial dependence of $a_{\mu}(x)$. Non-compatibility of a type, similar but not exactly the same as the one for the axial current mentioned above, again exists for the vector current. The calculations are all carried up to order $e^{2} g$, where $g$ is the fermion axial-vector coupling constant.

\section{ANOMALY OF THE DIVERGENCE OF THE AXIAL VECTOR CURRENT}

Although the topic in this section has been discussed by several authors $[2,4]$, we present it in a slightly different form which makes our discussion transparent. We also make a correspondence between the formal derivations and Feynman diagrams to emphasize that both lead to the same result. We define the axial vector current composed of charged fermion in the presence of the electromagnetic interaction as

$$
j_{\mu}^{5}=\lim _{\epsilon \rightarrow 0} j_{\mu}^{5 \epsilon}(x)
$$

with

$$
j_{\mu}^{5 \epsilon}(x)=i g T\left(\bar{\psi}\left(x+\frac{1}{2} \epsilon\right) \gamma_{\mu} \gamma_{5} \exp \left(i e \int_{-\frac{1}{2} \epsilon}^{\frac{1}{2} \epsilon} A_{\rho}(x+t) \mathrm{d} t \rho\right) \psi\left(x-\frac{1}{2} \epsilon\right)\right),
$$

where $T$ stand for the Wick's $T$ product, and the dependence of the current on the photon field is dictated by the gauge invariance. The limiting procedure in eq. (2) is defined as

$$
\lim _{\epsilon \rightarrow 0} \equiv \lim _{x(\epsilon) \rightarrow \delta^{4}(\epsilon)} \int d^{4} \epsilon x(\epsilon),
$$

that is, the procedure of averaging over four-dimensional $\epsilon$-space with a weight function which tends to $\delta$-function in the limit. This operation implies, in effect, the relations

$$
\begin{gathered}
\lim _{\epsilon \rightarrow 0} \epsilon_{\lambda} f\left(\epsilon^{2}\right)=\lim _{\epsilon \rightarrow 0} \epsilon_{\lambda} \epsilon_{\nu} \epsilon_{\rho} f\left(\epsilon^{2}\right)=0 \quad \text { etc. } \\
\lim _{\epsilon \rightarrow 0} \frac{\epsilon_{\lambda} \epsilon_{\nu}}{\epsilon^{2}}=\frac{1}{4} \delta_{\lambda \nu}
\end{gathered}
$$

The vector current is also defined in a similar way

$$
j_{\mu}^{\epsilon}(x)=i e \bar{\psi}\left(x+\frac{1}{2} \epsilon\right) \gamma_{\mu} \exp \left[i e \int_{-\frac{1}{2} \epsilon}^{\frac{1}{2} \epsilon} A_{\rho}\left(x+\frac{1}{2} \epsilon\right) \mathrm{d} t_{\rho}\right] \psi\left(x-\frac{1}{2} \epsilon\right)
$$


and satisfies the equation

$$
-\square A_{\mu}=j_{\mu}(x)=\lim _{\epsilon \rightarrow 0} j_{\mu}^{\epsilon}(x) .
$$

However the definition of the vector current with the extended space-time point will be irrelevant to the discussion in this section.

We calculate the matrix element of the axial vector current between a $2 \gamma$ state and the vacuum state,

$$
\left\langle k_{1}, k_{2}\left|j_{\mu}^{5}(0)\right| 0\right\rangle=e^{2} g \frac{e_{\lambda}^{(1)} e^{(2)}}{\sqrt{4 k_{10} k_{20}}} T_{\lambda \nu \mu}^{5}\left(k_{1}, k_{2}\right),
$$

along with that of the pseudoscalar density

$$
\left\langle k_{1}, k_{2}\left|j^{5}(0)\right| 0\right\rangle=e^{2} g \frac{e_{\lambda}^{(1)} e^{(2)}}{\sqrt{4 k_{10} 0_{20}}} T_{\left.\lambda \nu^{\left(k_{1}\right.}, k_{2}\right)}^{5},
$$

where $k_{1}, k_{2}$ and $e^{(1)}, e^{(2)}$ are momenta and polarization vectors of the two photons, respectively. The pseudoscalar density $j^{5}(x)$ is defined in a similar manner as in eqs. (1) and (2) except that the Dirac matrix $\gamma_{\mu} \gamma_{5}$ be replaced by $\gamma_{5}$.

The LSZ reduction method leads us to

$$
\begin{gathered}
e^{2} g T_{\lambda \nu \mu}^{5}\left(k_{1}, k_{2}\right)=\lim _{\epsilon \rightarrow 0} \int \mathrm{e}^{-i k_{1} x_{1}-i k_{2} x_{2}}\left\{\left\langle 0\left|T\left(j_{\lambda}\left(x_{1}\right) j_{\nu}\left(x_{2}\right) j_{\mu}^{5 \epsilon}(0)\right)\right| 0\right\rangle\right. \\
+\left\langle 0\left|T\left(\left[\dot{A}_{\lambda}\left(x_{1}\right)-i k_{10} A_{\lambda}\left(x_{1}\right), j_{\mu}^{5 \epsilon}(0)\right] \delta\left(x_{10}\right), j_{\nu}\left(x_{2}\right)\right)\right| 0\right\rangle \\
+\left\langle 0\left|\left(T\left[\dot{A}_{\nu}\left(x_{2}\right)-i k_{20} A_{\lambda}\left(x_{2}\right), j_{\mu}^{5 \epsilon}(0)\right] \delta\left(x_{20}\right), j_{\lambda}\left(x_{1}\right)\right)\right| 0\right\rangle \\
+\left\langle 0\left|T\left(\left[\dot{A}_{\nu}\left(x_{2}\right)-i k_{20} A_{\nu}\left(x_{2}\right), j_{\lambda}\left(x_{1}\right)\right] \delta\left(x_{10}-x_{20}\right), j_{\mu}^{5 \epsilon}(0)\right)\right| 0\right\rangle \\
+\left\langle 0\left|\left[\dot{A}_{\nu}\left(x_{2}\right)-i k_{20} A_{\nu}\left(x_{2}\right),\left[\dot{A}_{\lambda}\left(x_{1}\right)-i k_{10} A_{\lambda}\left(x_{1}\right), j_{\mu}^{5 \epsilon}(0)\right]\right] \delta\left(x_{10}\right) \delta\left(x_{20}\right)\right| 0\right\rangle .
\end{gathered}
$$

Here, since the $\epsilon$-limit associated with the axial vector current should be taken at the end, the $\epsilon$-limit in other currents is to be understood to be taken already without any harm. Then the fourth term in eq. (10) vanishes because it is proportional to the parameter $\epsilon$ associated with the vector current $j_{\lambda}\left(x_{1}\right)$. The last term also vanishes due to the trace calculation of the $\gamma$-matrices. The remaining three terms correspond to the three graphs of fig. 1 (a), (b) and (c), in the lowest order of perturbation calculation. The "bubble" graphs figs. 1 (b) and 1(c) are the new Feynman graphs introduced by the $\epsilon$-limiting process. The alternative possibility of a bubble graph with both the photons emitted at the same point is to be dropped because that would correspond to the fourth term in eq. (10) which as we have just pointed out vanishes because of the limiting procedure. 


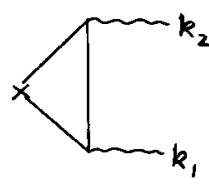

(a1)

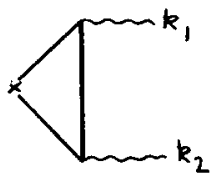

(a2)

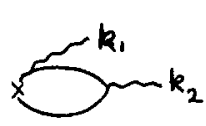

(b)

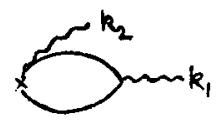

(c)

Fig. 1 .

Using the canonical commutation relations of the photon fields in the Lorentz gauge,

$$
\left[A_{\lambda}(x), A_{\nu}(y)\right] \delta\left(x_{0}-y_{0}\right)=i \delta_{\lambda \nu} \delta(y-x),
$$

we obtain the expressions for these graphs as

$$
T_{\lambda \nu \mu}^{5}\left(k_{1}, k_{2}\right)=T_{\lambda \nu \mu}^{5(\mathrm{a})}\left(k_{1}, k_{2}\right)+T_{\lambda \nu \mu}^{5(\mathrm{~b})}\left(k_{1}, k_{2}\right)+T_{\lambda \nu \mu}^{5(\mathrm{c})}\left(k_{1}, k_{2}\right),
$$

where

$$
\begin{aligned}
T_{\lambda \nu \mu}^{5(\mathrm{a})}=\lim _{\epsilon \rightarrow 0} & \frac{1}{(2 \pi)^{4}} \mathrm{e}^{\frac{1}{2} i \epsilon\left(k_{1}-k_{2}\right)} \int \mathrm{e}^{-i \epsilon p} \operatorname{Tr}\left(\gamma_{\mu} \gamma_{5} \frac{1}{i \gamma\left(p-k_{1}\right)+m}\right. \\
& \left.\quad \times \gamma_{\lambda} \frac{1}{i \gamma p+m} \gamma_{\nu} \frac{1}{i \gamma\left(p+k_{2}\right)+m}\right) \mathrm{d}^{4} p+\left(k_{1} \rightarrow k_{2}, \lambda \rightarrow \nu\right),
\end{aligned}
$$

$T_{\lambda \nu \mu}^{5(\mathrm{~b})}\left(k_{1}, k_{2}\right)$

$$
\begin{aligned}
& =\lim _{\epsilon \rightarrow 0} \frac{\epsilon_{\lambda}}{(2 \pi)^{4}} \mathrm{e}^{\frac{1}{2} i k_{2} \epsilon} \int \mathrm{e}^{-i p \epsilon} \operatorname{Tr}\left(\gamma_{\mu} \gamma_{5} \frac{1}{i \gamma\left(p-k_{1}\right)+m} \gamma_{\nu} \frac{1}{i \gamma p+m}\right) \mathrm{d}^{4} p \\
& =\lim _{\epsilon \rightarrow 0} \frac{1}{2 \pi^{2}} \epsilon^{\lambda \mu \rho \sigma} k_{2 \sigma} \frac{\epsilon_{\sigma} \epsilon}{\epsilon^{2}} \\
& =-\frac{1}{8 \pi^{2}} \epsilon^{\lambda \nu \mu \rho_{2 \rho}}
\end{aligned}
$$

$T_{\lambda \nu \mu}^{5(\mathrm{c})}\left(k_{1}, k_{2}\right)=T_{\nu \lambda \mu}^{5(\mathrm{~b})}\left(k_{2}, k_{1}\right)=\frac{1}{8 \pi^{2}} \epsilon^{\lambda \nu \mu \rho_{k}}{ }_{1 \rho}$.

It is also easy to derive the following relations 


$$
\begin{aligned}
& i k_{1 \lambda} T_{\lambda \nu \mu}^{5(\mathrm{a})}=\lim _{\epsilon \rightarrow 0} \frac{1}{(2 \pi)^{4}} \epsilon^{\frac{1}{2} i \epsilon\left(k_{1}-k_{2}\right)} \int \mathrm{e}^{-i \epsilon p} \\
& \left\{\operatorname{Tr}\left(\gamma_{\mu} \gamma_{5}\left[\frac{1}{i \gamma\left(p-k_{1}\right)+m}-\frac{1}{i \gamma p+m}\right] \gamma_{\nu} \frac{1}{i \gamma\left(p+k_{2}\right)+m}\right)\right. \\
& \left.+\operatorname{Tr}\left(\gamma_{\mu} \gamma_{5} \frac{1}{i \gamma\left(p-k_{2}\right)+m} \gamma_{\nu}\left[\frac{1}{i \gamma p+m}-\frac{1}{i \gamma\left(p+k_{1}\right)+m}\right]\right)\right\} \mathrm{d}^{4} p \\
& =\frac{i}{8 \pi^{2}} \epsilon^{\nu \mu \rho \sigma}{ }_{1 \rho} k_{2 \sigma} \text {, } \\
& -i\left(k_{1}+k_{2}\right) T_{\lambda \nu \mu}^{5(\mathrm{a})}=\lim _{\epsilon \rightarrow 0} \mathrm{e}^{\frac{1}{2} i \epsilon\left(k_{1}-k_{2}\right)} \int \mathrm{e}^{-i \epsilon p}\left\{2 m \operatorname { T r } \left(\gamma_{5} \frac{1}{\dot{i \gamma}\left(p-k_{1}\right)+m}\right.\right. \\
& \left.\left.\gamma_{\lambda} \frac{1}{i \gamma p+m} \gamma_{\nu} \frac{1}{i \gamma\left(p+k_{2}\right)+m}\right)\right\} \mathrm{d}^{4} p+\left(k_{1} \leftrightarrow k_{2}, \lambda \leftrightarrow \nu\right) \\
& -\lim _{\epsilon \rightarrow 0} \mathrm{e}^{\frac{1}{2} i \epsilon\left(k_{1}-k_{2}\right)} \int \mathrm{e}^{-i \epsilon p} \operatorname{Tr}\left(\gamma_{5} \gamma_{\lambda} \frac{1}{i \gamma p+m} \gamma_{\nu} \frac{1}{i \gamma\left(p+k_{2}\right)+m}\right. \\
& \left.\left.+\gamma_{5} \frac{1}{i \gamma\left(p-k_{1}\right)+m} \gamma_{\lambda} \frac{1}{i \gamma p+m} \gamma_{\nu}\right) \mathrm{d}^{4} p\right\}+\left(k_{1} \leftrightarrow k_{2}, \lambda \leftrightarrow \nu\right) \\
& =2 m T_{\lambda \nu}^{5}+\frac{i}{4 \pi^{2}} \epsilon^{\lambda \nu \rho \sigma} k_{1 \rho} k_{2 \sigma} .
\end{aligned}
$$

Note that the contributions from the equal time commutators as well as those from the surface terms are absent for the matrix element $T_{\lambda}^{5}$.

From eqs. (12) - (16), it follows that

$$
{ }^{i k}{ }_{1 \lambda} T_{\lambda \nu \mu}^{5}=i k_{2 \nu} T_{\lambda \nu \mu}^{5}=0
$$

and that

$$
-i\left(k_{1}+k_{2}\right){ }_{\mu}^{T} T_{\lambda \nu \mu}^{5}-2 m T_{\lambda \nu}^{5}=\frac{i}{2 \pi^{2}} \epsilon^{\lambda \nu \rho \sigma_{1 \rho}} k_{2 \sigma},
$$

i.e., that the gauge invariance is satisfied (eq. (17)) and that the Ward identity for the axial vector current is violated (eq. (18)). In the operator form, eq. (18) implies that

$$
{ }_{\mu} j_{\mu}^{5}(x)-2 m j^{5}(x)=i g \frac{e^{2}}{16 \pi^{2}} \widetilde{F}_{\mu \nu} F_{\mu \nu},
$$

where 


$$
\begin{aligned}
& \widetilde{F}_{\mu \nu}=\epsilon^{\mu \nu \rho \sigma_{F}}{ }_{\rho \sigma}, \\
& F_{\mu \nu}=\partial_{\mu} A_{\nu}-\partial_{\nu} A_{\mu} .
\end{aligned}
$$

The right hand side of eq. (18) is the anomaly pointed out by Adler [1].

It is clear from our method of derivation, that if we redefine the axialcurrent as

$$
\begin{gathered}
\bar{j}_{\mu}^{5}(x)=\lim _{\epsilon \rightarrow 0} T\left(\bar{\psi}\left(x+\frac{1}{2} \epsilon\right) \gamma_{\mu} \gamma_{5} \exp \left(-i e \int_{-\frac{1}{2} \epsilon}^{\frac{1}{2} \epsilon} A_{\rho}(x+t) \mathrm{d} t \rho\right) \psi\left(x-\frac{1}{2} \epsilon\right)\right. \\
-i g \frac{e^{2}}{8 \pi^{2}} \widetilde{F}_{\mu \nu} A_{\nu},
\end{gathered}
$$

then the current satisfies the PCAC condition

$$
\partial_{\mu} \bar{j}_{\mu}^{5}(x)=2 m j^{5}(x) \text {. }
$$

However, the transversality condition on the tensor $\bar{T}_{\lambda \nu \mu}^{5}\left(k_{1}, k_{2}\right)$ defined by

$$
\left\langle k_{1} k_{2}\left|\bar{j}_{\mu}^{5}(0)\right| 0\right\rangle=e^{2} g \frac{e_{\lambda}^{(1)} e_{\nu}^{(2)}}{\sqrt{4 k_{10} 0^{k} 20}} \bar{T}_{\lambda \nu \mu}^{5}\left(k_{1}, k_{2}\right)
$$

is no longer satisfied, i.e.,

$$
i k_{1 \lambda} \bar{T}_{\lambda \nu \mu}^{5} \neq 0, \quad i k_{2 \nu} \bar{T}_{\lambda \nu \mu}^{5} \neq 0 \text {. }
$$

The PCAC and the transversality conditions are thus mutually incompatible.

\section{DIVERGENCE OF THE VECTOR CURRENT IN THE PRESENCE OF AXIAL-VECTOR FIELD}

We discuss the matrix element of the vector current in a similar way, when there exists an axial vector field which satisfies the equation of motion

where

$$
-\partial_{\nu} G_{\nu \mu}+m_{a}^{2} a_{\mu}(x)=j_{\mu}^{5}(x)=\lim _{\epsilon \rightarrow 0} j_{\mu}^{5 \epsilon}(x),
$$

$$
\begin{gathered}
G_{\nu \mu}(x)=\partial_{\nu} a_{\mu}(x)-\partial{ }_{\mu} a_{\nu}(x), \\
j_{\mu}^{5 \epsilon}(x)=i g T\left(\overline { \psi } ( x + \frac { 1 } { 2 } \epsilon ) \gamma _ { \mu } \gamma _ { 5 } \operatorname { e x p } \left[i e \int_{-\frac{1}{2} \epsilon}^{\frac{1}{2} \epsilon} A_{\lambda}(x+t) \mathrm{d} t_{\lambda}+i g \eta \gamma_{5}\right.\right. \\
\left.\left.\times \int_{-\frac{1}{2} \epsilon}^{\frac{1}{2} \epsilon} a_{\lambda}(x+t) \mathrm{d} t_{\lambda}\right] \psi\left(x-\frac{1}{2} \epsilon\right)\right) .
\end{gathered}
$$


We also assume the canonical commutation relation

$$
\left[a_{k}(x), G_{\mathrm{o} l}(y)\right] \delta\left(x_{\mathrm{o}}-y_{\mathrm{o}}\right)=i \delta_{k l} \delta(x-y),
$$

as usual. The necessity of an axial-field dependence in the definition of the axial vector current has been discussed in ref. [3], which determined the coefficient $\eta$ to be 1 , from the requirement of consistency with the equation of motion and the Lorentz covariance. The vector current, therefore, may be modified as

$j_{\mu}^{\epsilon}(x)$

$=i e \bar{\psi}\left(x+\frac{1}{2} \epsilon\right) \gamma_{\mu} \exp \left[i e \int_{-\frac{1}{2} \epsilon}^{\frac{1}{2} \epsilon} A_{\lambda}(x+t) \mathrm{d} t_{\lambda}+i g \gamma_{5} \xi \int_{-\frac{1}{2} \epsilon}^{\frac{1}{2} \epsilon} a_{\lambda}(x+t) \mathrm{d} t_{\lambda}\right] \psi\left(x-\frac{1}{2} \epsilon\right)$,

where the value of the coefficient $\xi$ of the $a_{\lambda}$ dependent term in the exponent is to be discussed in this section.

3.1. Reciprocal relation between the field dependence of the currents

From the independence of the canonical variables

$$
\begin{aligned}
& {\left[G_{\mathrm{o} k}(x), \partial_{0} A_{l}(y)\right]_{x_{\mathrm{o}}=y_{\mathrm{o}}}=0,} \\
& {\left[a_{k}(x), A_{l}(y)\right]_{x_{\mathrm{o}}=y_{\mathrm{o}}}=\left[G_{\left.\mathrm{o} k^{(x)}, A_{l}(y)\right]_{x_{\mathrm{o}}=y_{\mathrm{o}}}}\right.} \\
& =\left[a_{k}(x), \partial_{\sigma} A_{l}(y)\right]_{x_{\mathrm{o}}=y_{\mathrm{o}}}=0,
\end{aligned}
$$

it follows that

$$
\left[\partial_{\mathrm{o}} G^{\mathrm{ok}}(x), \partial_{\mathrm{o}} A l(y)\right]_{x_{\mathrm{o}}=y_{\mathrm{o}}}=\left[\partial_{\mathrm{o}}^{2} A l(y), G^{\mathrm{ok}}(x)\right]_{x_{\mathrm{o}}=y_{\mathrm{o}}},
$$

which, upon using eqs. (7), (20) and (24), becomes

$$
\left[j_{k}^{5}(x), \partial_{\circ} A_{l}(y)\right]_{x_{\mathrm{o}}=y_{\mathrm{o}}}=\left[j_{l}(y), G_{\mathrm{o} k}(x)\right]_{x_{\mathrm{o}}=y_{\mathrm{o}}} .
$$

This is the space components of the covariant expression

$$
\frac{\delta j_{\mu}^{5}(x)}{\delta A_{\nu}(y)}=\frac{\delta j_{\nu}(y)}{\delta a_{\mu}(x)} .
$$

Eq. (27) is trivially satisfied as an integrability condition if the currents are derivable from a Lagrangian $L$ by the operations

$$
j_{\mu}(x)=\frac{\delta L}{\delta A_{\mu}(x)}
$$




$$
j_{\mu}^{5}(y)=\frac{\delta L}{\delta a_{\mu}(x)}
$$

Since our equations of motion (7) and (20) are not derived from a Lagrangian excep̣t in the limit $\epsilon \rightarrow 0$, eq. (26) or eq. (27) leads to a non-trivial restriction on the dependence of the currents on the fields. In fact, by using eq. (27) we obtain

$$
\begin{aligned}
& M_{\mu \nu} \equiv \lim _{\epsilon \rightarrow 0} T\left(\bar{\psi}\left(\frac{1}{2} \epsilon \gamma_{\mu} \gamma_{5} \epsilon_{\nu} \exp \left[i e \int_{-\frac{1}{2} \epsilon}^{\frac{1}{2} \epsilon} A_{\lambda} \mathrm{d} t_{\lambda}+i g \gamma_{5} \int_{-\frac{1}{2} \epsilon}^{\frac{1}{2} \epsilon} a_{\lambda} \mathrm{d} t_{\lambda}\right] \psi\left(-\frac{1}{2} \epsilon\right)\right)\right. \\
& =\xi \lim _{\epsilon \rightarrow 0} T\left(\bar{\psi}\left(\frac{1}{2} \epsilon\right) \gamma_{\nu} \gamma_{5} \epsilon{ }_{\mu} \exp \left[i e \int_{-\frac{1}{2} \epsilon}^{\frac{1}{2} \epsilon} A_{\lambda} \mathrm{d} t_{\lambda}+i g \gamma_{5} \xi \int_{-\frac{1}{2} \epsilon}^{\frac{1}{2} \epsilon} a_{\lambda} \mathrm{d} t_{\lambda}\right] \psi\left(-\frac{1}{2} \epsilon\right) .\right.
\end{aligned}
$$

The matrix element of eq. (29) between one photon state and the vacuum state can be easily calculated (noting that the equal time commutator in the LSZ reduction vanishes in this case):

$$
\begin{aligned}
\left\langle k\left|M_{\mu \nu}\right| 0\right\rangle & =i \frac{e_{\lambda}}{\sqrt{2 k_{\mathrm{o}}}} \int \mathrm{e}^{-i k x}\left\langle 0\left|T\left(j_{\lambda}(x), M_{\mu \nu}\right)\right| 0\right\rangle \\
& =\frac{-e e_{\lambda}}{\sqrt{2 k_{\mathrm{o}}}} T_{\lambda \nu \mu}^{5(\mathrm{~b})}(\mathrm{o}, k)=\frac{e e_{\lambda}}{\sqrt{2 k_{\mathrm{o}}}} \frac{1}{8 \pi^{2}} \epsilon^{\lambda \nu \mu \rho_{k}}{ }_{k_{\rho}} .
\end{aligned}
$$

Hence, eq. (29) reads

$$
M_{\mu \nu}=\xi M_{\nu \mu}=-\xi_{\mu \nu}
$$

from which we conclude that

$$
\xi=-1 \text {. }
$$

It should be noted that the requirement of the second kind of $\gamma_{5}$ gauge transformation leads us to the parameter $\xi=+1$ as in the case of the coefficient $\eta$ of the $a_{\lambda}$ dependence in the axial vector current, in contradistinction to the choice (32).

\subsection{Matrix element of the vector current}

We next calculate the matrix element $\left\langle k\left|j_{\lambda}(0)\right| q\right\rangle$, where the states $(k)$ and $(q)$ are those of one photon and one axial vector field. Proceeding as in sect. 2 , we have

$$
\begin{gathered}
\left\langle k\left|j_{\lambda}(0)\right| q\right\rangle=e^{2} g \frac{e_{\nu} e_{\mu}^{5}}{\sqrt{4 k_{\mathrm{o}} q_{\mathrm{o}}}} T_{\lambda \nu \mu}(k, q), \\
T_{\lambda \nu \mu}=T_{\lambda \nu \mu}^{(\mathrm{a})}+T_{\lambda \nu \mu}^{(\mathrm{b})}+T_{\lambda \nu \mu}^{(\mathrm{c})},
\end{gathered}
$$


where

$$
\begin{aligned}
& T_{\lambda \nu \mu}^{(\mathrm{a})}(k, q)=\lim _{\epsilon \rightarrow 0} \frac{1}{e^{2} g} \int \mathrm{e}^{-i k x+i q y}\left\langle 0\left|T\left(j_{\nu}(x) j_{\mu}^{5}(y) j_{\lambda}^{\epsilon}(0)\right)\right| 0\right\rangle \mathrm{d}^{4} x \mathrm{~d}^{4} y \\
&=\lim _{\epsilon \rightarrow 0} \frac{\mathrm{e}^{\frac{1}{2} i \epsilon s}}{(2 \pi)^{4}} \int \mathrm{e}^{-i p \epsilon} \operatorname{Tr}\left[\gamma_{\mu} \gamma_{5} \frac{1}{i \gamma(p-s)+m} \gamma_{\lambda} \frac{1}{i \gamma p+m} \gamma_{\nu}\right. \\
&+\lim _{\epsilon \rightarrow 0} \frac{\mathrm{e}^{-\frac{1}{2} i \epsilon s}}{(2 \pi)^{4}} \int \mathrm{e}^{-i p \epsilon} \operatorname{Tr}\left[\gamma_{\mu} \gamma_{5} \frac{1}{i \gamma(p-k)+m} \gamma_{\nu} \frac{1}{i \gamma p+m} \gamma_{\lambda} \frac{1}{i \gamma(p+s)+m}\right] \mathrm{d}^{4} p
\end{aligned}
$$

$$
\begin{aligned}
T_{\lambda \nu \mu}^{(\mathrm{b})}(k, q) & =\lim _{\epsilon \rightarrow 0} \frac{i g \xi \epsilon}{e^{2} g} \int \mathrm{e}^{-i k x+i q y} \delta(y)\left\langle 0\left|T\left(j_{\lambda}^{5 \epsilon}(0) j_{\nu}(x)\right)\right| 0\right\rangle \mathrm{d}^{4} x \mathrm{~d}^{4} y \\
& =\lim _{\epsilon \rightarrow 0} \frac{\mathrm{e}^{\frac{1}{2} i k \epsilon}}{(2 \pi)^{4}} \epsilon_{\mu}^{\xi \int \mathrm{e}^{-i p \epsilon}} \operatorname{Tr}\left(\gamma_{\lambda} \gamma_{5} \frac{1}{i \gamma(p-k)+m} \gamma_{\nu} \frac{1}{i \gamma p+m}\right) \mathrm{d}^{4} p \\
& =\frac{\xi}{8 \pi^{2}} \epsilon^{\lambda \nu \mu \rho} k_{\rho},
\end{aligned}
$$

$$
\begin{aligned}
T_{\lambda \nu \mu}^{(c)}= & \lim _{\epsilon \rightarrow 0} \frac{1}{e^{2} g} \int \mathrm{e}^{-i k x+i q y}\langle 0| T\left(\left[A_{\nu}-i k_{\mathrm{o}} A_{\nu}(x), j_{\lambda}^{\epsilon}(0)\right]\right. \\
& \left.\quad \times \delta\left(x_{\mathrm{o}}\right), j_{\mu}^{5}(y)\right)|0\rangle \mathrm{d}^{4} x \mathrm{~d}^{4} y \\
= & \lim _{\epsilon \rightarrow 0} \frac{\mathrm{e}^{\frac{1}{2} i q \epsilon}}{(2 \pi)^{4}} \epsilon_{\nu} \int \mathrm{e}^{-i p \epsilon} \operatorname{Tr}\left(\gamma_{\mu} \gamma_{5} \frac{1}{i \gamma(p-q)+m} \gamma_{\lambda} \frac{1}{i \gamma p+m}\right) \mathrm{d}^{4} p \\
= & \frac{1}{8 \pi^{2}} \epsilon^{\lambda \nu \mu \rho} q_{\rho} .
\end{aligned}
$$

In eq. (35), we have used a notation

$$
s=q-k,
$$

and the three terms in eq. (34) correspond to the graphs in fig. 2, except that the term that contains $\partial_{\mu} \partial_{\rho} a_{\rho}$ in the LSZ reduction formula is cancelled by a part of the equal time commutator of diagrams 2(b) and 2(c), as is explained in appendix 1.

In order to examine various Ward Identities, we make contractions of eq. (35) with momenta as before, obtaining, 


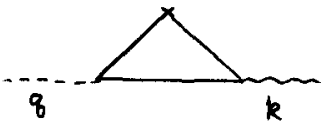

(a1)

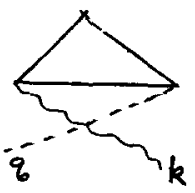

(a2)

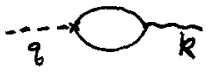

(b)

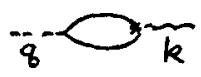

(c)

Fig. 2.

$$
\begin{aligned}
i k_{\nu} T_{\lambda \nu \mu}^{(\mathrm{a})}=\lim _{\epsilon \rightarrow 0} \frac{\mathrm{e}^{\frac{1}{2} i \epsilon s}}{(2 \pi)^{4}} \int \mathrm{e}^{-i p \epsilon} \operatorname{Tr}\left[\gamma_{\mu} \gamma_{5} \frac{1}{i \gamma(p-s)+m} \gamma_{\lambda}\right. \\
\left.\quad \times\left(\frac{1}{i \gamma p+m}-\frac{1}{i \gamma(p+k)+m}\right)\right] \mathrm{d}^{4} p \\
+\lim _{\epsilon \rightarrow 0} \frac{\mathrm{e}^{-\frac{1}{2} i \epsilon s}}{(2 \pi)^{4}} \int \mathrm{e}^{-i p \epsilon} \operatorname{Tr}\left[\gamma_{\mu} \gamma_{5}\left(\frac{1}{i \gamma(p-k)+m}-\frac{1}{i \gamma p+m}\right) \gamma_{\lambda} \frac{1}{i \gamma(p+s)+m}\right] \mathrm{d}^{4} p \\
=-\frac{1}{8 \pi^{2}} \epsilon^{\lambda \mu \rho \sigma} q_{\rho}{ }^{k},
\end{aligned}
$$

and similarly

$$
i(q-k)_{\lambda} T_{\lambda \nu \mu}^{(\mathrm{a})}=\frac{i}{4 \pi^{2}} \epsilon^{\nu \mu \rho \sigma} q_{\rho}{ }^{k}{ }_{\sigma} .
$$

Combining eqs. (36) $-(39)$, we get

$$
\begin{gathered}
i k_{\nu} T_{\lambda \nu \mu}=0, \\
i(q-k)_{\lambda} T_{\lambda \nu \mu}=\frac{i}{8 \pi^{2}}(3+\xi) \epsilon^{\nu \mu \rho \sigma} q_{\rho} k_{\sigma} .
\end{gathered}
$$

While eq. (40) guarantees the gauge invariance with respect to the external photon, eq. (41) tells us that the conservation law of the vector current defined in eqs. (6') and (7) is not satisfied unless

$$
\xi=-3 \text {. }
$$

If we use the value of the parameter $\xi$ that has been derived in the preceeding subsect., eq. (32), we are led to an operator equation

$$
\partial_{\mu} j_{\mu}(x)=\frac{i e^{2} g}{16 \pi^{2}} \tilde{F}_{\mu \nu} G_{\mu \nu},
$$

the right hand side of which is an anomaly similar to that of eq. (18). How- 
ever, in the subsect. 3.3, we present another argument which leads to eq. (42), so that we have a contradiction for the determination of $\xi$.

\subsection{Commutativity of the differential operators}

We consider the following identity

$$
\begin{gathered}
I_{\lambda \nu} \equiv e_{\mu}^{5} \int \mathrm{e}^{-i k_{1} x_{1}-i k_{2} x_{2}+i q y}\left(-\square_{x_{1}}\right)\left(-\square_{x_{2}}\right)\left(-\square_{y}+m_{\mathrm{a}}^{2}\right) \\
\times\left\langle 0\left|T\left(A_{\nu}\left(x_{1}\right) A_{\nu}\left(x_{2}\right) a_{\mu}(y)\right)\right| 0\right\rangle \mathrm{d}^{4} x_{1} \mathrm{~d}^{4} x_{2} \mathrm{~d}^{4} y, \\
=J_{\lambda \nu} \equiv e_{\mu}^{5} \int \mathrm{e}^{-i k_{1} x_{1}-i k_{2} x_{2}+i g_{y}}\left(-\square_{y}+m_{\mathrm{a}}^{2}\right)\left(-\square_{x_{2}}\right)\left(-\square_{x_{1}}\right) \\
\times\left\langle 0\left|T\left(v_{\lambda}\left(x_{1}\right) v_{\nu}\left(x_{2}\right) a_{\mu}(y)\right)\right| 0\right\rangle \mathrm{d}^{4} x_{1} \mathrm{~d}^{4} x_{2} \mathrm{~d}^{4} y,
\end{gathered}
$$

where the three momenta are taken on mass shell.

By making the differentiation successively, and using the result of appendix 1 , it is easy to derive the relations

$$
\begin{aligned}
& I_{\lambda \nu}=e^{2} g(2 \pi)^{4} \delta\left(q-k_{1}-k_{2}\right) e_{\mu}^{5} T_{\lambda \nu \mu}^{5}\left(k_{1}, k_{2}\right), \\
& J_{\lambda \nu}=e^{2} g(2 \pi)^{4} \delta\left(q-k_{1}-k_{2}\right) e_{\mu}^{5} T_{\lambda \nu \mu}\left(k_{2}, q\right),
\end{aligned}
$$

where $T_{\lambda \nu \mu}^{5}$ and $T_{\lambda \nu \mu}$ are defined in eq. (10) and eq. (33) respectively. Then, from eq. (17) and eq. (41), it follows that

$$
\begin{aligned}
i k_{1 \lambda} I_{\lambda \nu} e_{\nu} & =0 \\
& =i k_{1 \lambda}{ }^{J} \lambda \nu_{\nu}^{e} \\
& =i e^{2} g 2 \pi^{2} \delta\left(q-k_{1}-k_{2}\right)(3+\xi) e_{\mu}^{5} e_{\nu} \epsilon^{\nu \mu \rho \sigma_{q} k_{2 \sigma}},
\end{aligned}
$$

which again gives $\xi=-3$.

\section{DISCUSSION}

We have thus observed that the vector (axial-vector) current not only has a dependence on the field to which it is coupled to, but also on the axial-vector (vector) field. If the dependence is taken as dictated by the reciprocity relation, (which we believe is quite general) and the transversatility condition on the matrix elements of $j_{\mu}^{5}(0)$, then we are led to a non-conservation equation for the vector current very similar to the one obtained earlier for the axial-vector current. Alternatively, if one forces the conservation equation for the vector current, one has, through the reciprocity relations, a violation of the transversality condition on the matrix element of the axial-vector current. 
We conclude, therefore, that the way we introduced the extension of the currents is not compatible with the canonical quantization of vector (axialvector) fields to which the currents couple. As a matter of fact, our equations of motion defined in eqs. (7) and (20) do not correspond to any Lagrangian, which could be the source of the trouble.

If we start with a Lagrangian, we have two possibilities of formulating our problem.

(i) Extension of the interaction Lagrangian. In this case the Lagrangian can be written as

$$
L=L_{\mathrm{O}^{+}} \lim _{\epsilon \rightarrow 0} L_{I^{(}}(\epsilon),
$$

where the free Lagrangian $L_{\mathrm{o}}$ does not involve the extension by $\epsilon$-method. While the canonical quantization in this method is the same as usual, we lose the second kind of gauge invariance for the electromagnetic interactions. Although the reciprocity relation which was discussed in the preceeding section is a trivial matter, the source current is not gauge invariant, in general*. One is thus not assured of the transversality condition on the matrix elements.

(ii) The extended definition of the whole Lagrangian

$$
L=\lim _{\epsilon \rightarrow 0}\left(L_{\mathbf{o}}(\epsilon)+L_{I}(\epsilon)\right) .
$$

In this case, the gauge invariance can be satisfied very easily. However, the separation of the free and the interaction Lagrangian becomes difficult, and the canonical quantization method does not seem to be unique $[5,6]^{* *}$.

* The Lagrangian continues to be gauge invariant of the first kind, which in a naive approach is sufficient to guarantee conservation (transversality) of the vector current or PCAC for the axial-current. This however is no longer true in the formalism of the extended source with the $\epsilon$-limiting procedure indicated in eq. (48), since Noether's theorem no longer follows from the invariance requirement.

** If we follow the most primitive form of the canonical quantization, we cannot get a finite answer for the matrix elements discussed in this text, since the factor due to $\epsilon$ is absorbed in the definition of the canonical variables. For reasons of canonical quantization, one may prefer to use space-like spreading, i.e., $\epsilon=(\varepsilon, 0)$. This indeed has been considered by e.g. ref [5]. (In the latter work, spreading of the free Lagrangian $L_{\mathrm{o}}$ has been attempted to order $\varepsilon \cdot \nabla$ to define current. It seems, however, doubtful to us that this is a valid step, since the singular nature of the differential operator $\nabla$, when acting on Green's functions, may just compensate the small parameter $\epsilon$. If so, then all powers of $(\varepsilon \cdot \nabla)^{n}$ contribute.) It is also unclear how a covariant calculation procedure, such as for the current current correlation function, can be devised in this non-covariant context. Recently the difficulties of the $\epsilon-1$ imiting method has been discussed in ref. [6]. 


\section{APPENDIX}

LSZ reduction formula for a massive vector (or axial-vector) field which couples with a non-conserving current.

Let us consider the reduction of the axial vector field with momentum $q$ and the polarization vector $e_{\mu}$ in the matrix element out $\langle\alpha|\eta(X)| \beta, q\rangle_{\text {in }}$, where $\alpha, \beta$ are arbitrary asymptotic states and

$$
\eta(X) \equiv \eta\left(x_{1}, x_{2}, \ldots, x_{n}\right)=T\left(\varphi^{(1)}\left(x_{1}\right), \varphi^{(2)}\left(x_{2}\right), \ldots, \varphi^{(n)}\left(x_{n}\right)\right),
$$

$\varphi^{(i)}\left(x_{i}\right)$ being arbitrary local operators. We use the following shorthand convention,

$$
\begin{aligned}
& {[A(y), \eta(X)] \delta\left(y_{\mathrm{o}}-X_{\mathrm{o}}\right)} \\
& \quad \equiv \sum_{i=1}^{n} T\left(\varphi^{(1)}\left(x_{1}\right), \ldots,\left[A(y), \varphi^{(i)}\left(x_{i}\right)\right], \ldots, \varphi^{(n)}\left(x_{n}\right)\right) \delta\left(y_{\mathrm{o}}-x_{i \mathrm{o}}\right),
\end{aligned}
$$

and assume for simplicity that the states $\alpha, \beta$ do not contain the axial-vector field in question. Then, the LSZ reduction technique gives (dropping the in, out suffixes)

$$
\begin{aligned}
\langle\alpha|\eta(X)| \beta, q\rangle & =i \frac{e_{\mu}}{\sqrt{2 q_{\mathrm{o}}}} \int \mathrm{d}^{4} y \mathrm{e}^{i q y_{1}}\left\{\left\langle\alpha\left|T\left(\left(-\square+m_{\mathrm{a}}^{2}\right) a_{\mu}(y), \eta(X)\right)\right| \beta\right\rangle\right. \\
\left.+\left\langle 0\left|\left[\dot{a}_{\mu}(y)+i q_{\mathrm{o}} a_{\mu}(y), \eta(X)\right] \delta\left(y_{\mathrm{o}}-X_{\mathrm{o}}\right)\right| \beta\right\rangle\right\} & \\
= & \frac{i e_{\mu}}{\sqrt{2 q_{\mathrm{o}}}} \int \mathrm{d}^{4} y \mathrm{e}^{i q y}\left\{\left\langle\alpha\left|T\left(j_{\mu}^{5}(y), \eta(X)\right)\right| \beta\right\rangle\right. \\
-\langle\alpha| & T\left(\partial_{\mu}{ }^{\partial} a_{\rho}(y), \eta(X)\right)|\beta\rangle+\left\langle\alpha\left|\left[\dot{a}_{\mu}(y)+i q_{\mathrm{o}} a_{\mu}(y), \eta(X)\right] \delta\left(y_{\mathrm{o}}-X_{\mathrm{o}}\right)\right| \beta\right\rangle \\
& =I_{1}+I_{2}+I_{3} .
\end{aligned}
$$

The second term of the last expression can be expressed, by doing the partial integration, as

$$
\begin{aligned}
I_{2}=-\frac{i e_{\mu}}{\sqrt{2 q_{\mathrm{o}}}} \int \mathrm{d}^{4} y & \mathrm{e}^{i q y} \frac{\partial}{\partial y_{\mu}}\left\langle\alpha\left|T\left(\partial \rho a_{\rho}(y), \eta(X)\right)\right| \beta\right\rangle \\
& +\frac{e_{4}}{\sqrt{2 q_{\mathrm{o}}}} \int \mathrm{d}^{4} y \mathrm{e}^{i q y}\left\langle\alpha\left|\left[\partial_{\rho} a_{\rho}(y), \eta(X)\right] \delta\left(y_{\mathrm{o}}-X_{\mathrm{o}}\right)\right| \beta\right\rangle,
\end{aligned}
$$

where the first term vanishes because of the transversality condition for the physical particle 


$$
e_{\mu} q_{\mu}=0 .
$$

Combining the two terms $I_{2}$ and $I_{3}$, we obtain

$$
\begin{aligned}
I_{2}+I_{3}= & \frac{e_{4}}{\sqrt{2 q_{\mathrm{o}}}} \int \mathrm{d}^{4} y \mathrm{e}^{i q y}\left\langle\alpha\left|\left[\partial_{\rho} a_{\rho}(y)+i \dot{a}_{4}(y)-q_{\mathrm{o}} a_{4}(y), \eta(X)\right] \delta\left(y_{\mathrm{o}}-X_{\mathrm{o}}\right)\right| \beta\right\rangle \\
& +\frac{i e_{k}}{\sqrt{2 q_{\mathrm{o}}}} \int \mathrm{d}^{4} y \mathrm{e}^{i q y}\left\langle\alpha\left|\left[\dot{a}_{k}(y)+\partial_{k} a_{\mathrm{o}}+i q_{\mathrm{o}} a_{k}(y)-\partial_{k} a_{\mathrm{o}}, \eta(X)\right] \delta\left(y_{\mathrm{O}}-X_{\mathrm{o}}\right)\right| \beta\right\rangle \\
= & i \frac{\left(-e_{4} q_{k}+e_{k} q_{4}\right)}{\sqrt{2 q_{\mathrm{o}}}} \int \mathrm{d}^{4} y \mathrm{e}^{i q y}\left\langle\alpha\left|\left[a_{k}(y), \eta(X)\right] \delta\left(y_{\mathrm{o}}-X_{\mathrm{o}}\right)\right| \beta\right\rangle \\
& +i \frac{e_{k}}{\sqrt{2 q_{\mathrm{o}}}} \int \mathrm{d}^{4} y \mathrm{e}^{i q y}\left\langle\alpha\left|\left[G^{\mathrm{o} k}(y), \eta(X)\right] \delta\left(y_{\mathrm{o}}-X_{\mathrm{o}}\right)\right| \beta\right\rangle \\
& -\frac{(e q)}{\sqrt{2 q_{\mathrm{o}}}} \int \mathrm{d}^{4} y \mathrm{e}^{i q y}\left\langle\alpha\left|\left[a_{\mathrm{o}}(y), \eta(X)\right] \delta\left(y_{\mathrm{O}}-X_{\mathrm{O}}\right)\right| \beta\right\rangle
\end{aligned}
$$

The last term vanishes by transversality condition (A.5). The final expression for the LSZ reduction is, therefore, given by

$$
\begin{aligned}
&\langle\alpha|\eta(X)| \beta, q\rangle=\frac{i e_{\mu}}{\sqrt{2 q_{\mathrm{o}}}} \int \mathrm{d}^{4} y \mathrm{e}^{i q y}\left\langle\alpha\left|T\left(j_{\mu}^{5}(y), \eta(X)\right)\right| \beta\right\rangle \\
&+i \frac{e_{k}}{\sqrt{2 q_{\mathrm{o}}}} \int \mathrm{d}^{4} y \mathrm{e}^{i q y}\left\langle\alpha\left|\left[G^{\mathrm{o} k}(y), \eta(X)\right] \delta\left(y_{\mathrm{o}}-X_{\mathrm{o}}\right)\right| \beta\right\rangle \\
&+i \frac{\left(-e_{4} q_{k}+e_{k} q_{4}\right)}{\sqrt{2 q_{\mathrm{o}}}} \int \mathrm{d}^{4} y \mathrm{e}^{i q y}\left\langle\alpha\left|\left[a_{k}(y), \eta(X)\right] \delta\left(y_{\mathrm{o}}-X_{\mathrm{o}}\right)\right| \beta\right\rangle .
\end{aligned}
$$

It should be noticed that the equal time commutator contains only the canonical variables $a_{k}, G^{\mathrm{o} k}$, so that independent fields such as fermion field etc. in $\eta(X)$ does not contribute to the equal time commutators.

For $\eta=\epsilon_{\mu} a_{\mu}(x)$, the equal time commutator is calculated as 


$$
\begin{aligned}
e_{k}\left[G^{\mathrm{ok}}(y), \epsilon a(x)\right]+ & \left(-e_{4} q_{k}+e_{k} q_{4}\right)\left[a_{k}, \epsilon a\right] \\
& =e_{k}\left[-i \epsilon_{k} \delta(x-y)-\left[G^{\mathrm{o} k}, a^{0}\right] \epsilon^{0}\right]-i\left(-e_{\mathrm{o}} q_{k}+e_{k} q_{\mathrm{o}}\right)\left[a_{k}, a_{\mathrm{o}}\right] \epsilon_{\mathrm{o}} \\
& =-i e_{k} \epsilon_{k} \delta(y-x)-i \epsilon_{\mathrm{o}}\left(-e_{\mathrm{o}} q_{k}+e_{k} q_{\mathrm{o}}\right) \frac{i \partial_{k}}{m_{\mathrm{a}}^{2}} \delta(y-x) \\
& \Rightarrow-i e_{k} \epsilon_{k} \delta(y-x)-i \epsilon_{\mathrm{o}}\left\{-e_{\mathrm{o}}\left(\frac{q_{\mathrm{o}}^{2}}{\left.m^{2}-1\right)}\right)+e_{k} q_{\mathrm{o}} \frac{q_{k}}{m_{\mathrm{a}}^{2}}\right\} \delta(y-x) \\
& =-i\left\{(e \epsilon)+\epsilon_{\mathrm{o}} \frac{q_{\mathrm{o}}}{m_{\mathrm{a}}^{2}}(e q)\right\} \delta(y-x),
\end{aligned}
$$

in the lowest order in $\epsilon$ (or in the coupling constant). From this, we obtain the following formula which has been used in the text.

$$
\begin{aligned}
& \left\langle\alpha\left|j_{\lambda}^{\epsilon}(0)\right| \beta, q\right\rangle=\frac{i e_{\mu}}{\sqrt{2 q_{\mathrm{o}}}} \int \mathrm{d}^{4} y \mathrm{e}^{i q y}\left\langle\alpha\left|T\left(j_{\mu}^{5}(y), j^{\epsilon}(0)\right)\right| \beta\right\rangle \\
& \quad+i g \frac{\xi\left(e_{\mu} \epsilon_{\mu}\right)}{\sqrt{2 q_{\mathrm{o}}}}\left\langle\alpha\left|i e \bar{\psi}\left(\frac{1}{2} \epsilon\right) \gamma_{\lambda} \gamma_{5} \exp \left[i e \int A_{\rho} \mathrm{d} t_{\rho}+i g \xi \int a_{\rho} \mathrm{d} t_{\rho}\right] \psi\left(\frac{1}{2} \epsilon\right)\right| \beta\right\rangle
\end{aligned}
$$

\section{REFERENCES}

[1] S. L. Adler, Phys. Rev. 177 (1969) 2426.

[2] C. R. Hagen, Rochester preprint (1969); Phys. Rev. (in press).

[3] D. Boulware and S. Deser, Phys. Rev. 151 (1966) 1278.

[4] R. Jackiw and K. Johnson, Harvard preprint (1969).

[5] D. G. Boulware, Phys. Rev. 151 (1966) 1278; $J$. Trubatch, Nuovo Cimento 60A (1969) 239.

[6] R. C. Hagen, Non-existence of anomalous commutators in spinor electrodynamies, Rochester preprint (1969). 